\title{
Probabilistic Insurance
}

\author{
PETER P. WAKKER \\ CentER, Tilburg University, Tilburg, The Netherlands and Medical Decision Making Unit, Leiden University, \\ Leiden,The Netherlands,Wakker@MDM.MedFac.LeidenUniv.nl
}

\section{RICHARD H. THALER}

Graduate School of Business, University of Chicago, Chicago, IL 60637, USA and National Bureau of

Economic Research, Cambridge, MA 02139

\author{
AMOS TVERSKY \\ Stanford University, Stanford, CA 94305
}

\begin{abstract}
Probabilistic insurance is an insurance policy involving a small probability that the consumer will not be reimbursed. Survey data suggest that people dislike probabilistic insurance and demand more than a $20 \%$ reduction in the premium to compensate for a $1 \%$ default risk. While these preferences are intuitively appealing they are difficult to reconcile with expected utility theory. Under highly plausible assumptions about the utility function, willingness to pay for probabilistic insurance should be very close to willingness to pay for standard insurance less the default risk. However, the reluctance to buy probabilistic insurance is predicted by the weighting function of prospect theory. This finding highlights the potential role of the weighting function to explain insurance.
\end{abstract}

Key words: probabilistic insurance, decision weights, prospect theory

JEL Classification: D81

Insurance is a contract in which an individual pays a fixed premium and is promised to be paid in the event that a specific hazard occurs. Kahneman and Tversky (1979) introduced the notion of "probabilistic insurance" (PI), namely an insurance policy which, in the event that the hazard occurs, pays off with some probability strictly less than one. They showed, for a particular type of probabilistic insurance, that while consumers find such policies unattractive, an expected utility maximizer would actually prefer the probabilistic policy (at an appropriately reduced premium) to a policy that pays off with certainty. They also showed that the aversion to PI is consistent with prospect theory. Later, Segal (1988) showed that the rejection of PI (of the original Kahneman \& Tversky type) is also consistent with rank-dependent utility theory.

Probabilistic insurance seems like a novel concept, but most insurance policies are, in fact, probabilistic. Insurance policies typically specify some events (e.g., wars, "acts of god," contributory negligence) in which the consumer is not reimbursed for losses, whether or not the consumer was aware of these contingencies ex ante. Furthermore, there 
is always a possibility - however remote - that the insurer will not pay for some other reason such as insolvency or fraud. Although such default risks are not explicitly acknowledged, they are present in any real insurance setting.

In light of the insight that all insurance is essentially probabilistic, we provide here a more thorough account of the topic, from both descriptive and normative perspectives. To do so, we analyze a different type of PI than the one originally addressed by Kahneman and Tversky. The original version of Pl was selected for study because it was analytically tractable and led to the surprising result that a risk averse expected utility maximizer favors probabilistic over standard insurance. However, it has the special feature that in the event that the claim was not paid, the premium would be refunded. This contingency does not adequately capture the risk of default or fraud because in these instances a premium refund might be problematic. In this article we investigate a more natural form of PI that does not involve refunding of premia. We also discuss the issue of consumer surplus that was not addressed in the original treatment.

Section 1 presents survey data which reveal that people also dislike the revised version of PI. Section 2 shows that, under expected utility with very plausible assumptions about the utility function, the reservation price for probabilistic insurance is very close to the actuarially adjusted reservation price for standard insurance. Section 3 shows that the observed discrepancy between the reservation price for probabilistic insurance and the actuarial adjustment of the reservation price of standard insurance can be explained by the weighting function of prospect theory. The implications of these results to the analysis of risk bearing are discussed in Section 4. Proofs are presented in the appendix.

\section{Survey data}

Probabilistic insurance is intuitively unappealing. This intuition is shared by Robert Merton (1993) who observes that stellar credit ratings for investment banking firms issuing derivative securities are very important because investors are loathe to bear the risk of the bank's insolvency. As he put it: "Even if the insurance company offers an actuarially fair reduction in the price of the insurance, to reflect the risk of insolvency, a risk averse customer would prefer the policy with the least default risk. Indeed, on introspection, I doubt that many real world customers would consciously agree to accept non-trivial risk on a $\$ 200,000$ life insurance policy in return for a large reduction in the annual premium, say from $\$ 400$ to $\$ 300 ”$ (p. 43).

To confirm that Merton and we are not alone in thinking that PI is unattractive, we have conducted some surveys of various groups (students, executives, portfolio managers, etc.). Necessarily, these surveys are hypothetical. Though one could devise similar experiments for real money, the stakes would, of course, have to be affordably low. We believe that in this domain, thought experiments for large sums can be more instructive than real experiments for pennies. As the results show, the subjects share our (and Merton's) intuitions quite strongly.

The first question was given to a group of Stanford students $(N=86)$. It illustrates the problems we investigate below. 
(A). Imagine that you have graduated from college, you hold a job, and you own a small house. Assume that there is a risk of 1 in 200 per year (i.e., 1/2 of 1\%) that your house will catch fire. The full replacement cost of the house is $\$ 125,000$.

1. What is the most you would be willing to pay (per year) for an insurance policy that will cover all damages due to fire? $\$$ -

Median response: $\$ 700$.

2. Imagine that you have been offered a different kind of policy called probabilistic fire insurance. This policy is identical to the previous one except that there is $1 \%$ chance that in the event of a fire, your claim will not be paid. You can think about the risk this way: in case of fire, the insurance company will draw a 2-digit number at random, and if it matches the last 2 digits of your social security number, your costs will not be covered. What is the most you would be willing to pay (per year) for probabilistic fire insurance? \$-

Median response: $\$ 500$.

(B). Now suppose that the replacement cost of your house is $\$ 250,000$ (instead of $\$ 125,000)$, but your total wealth remains unchanged. That is, the house now represents a larger portion of your total wealth. Assume the risk remains the same ( 1 in 200 per year).

3. What is the most you would be willing to pay (per year) for an insurance policy that will cover all damages due to fire in this case? $\$$ -

\section{Median response: $\$ 1300$.}

4. What is the most you would be willing to pay for probabilistic fire insurance in this case? \$-

\section{Median response: $\$ 900$.}

These data indicate that probabilistic insurance is relatively unattractive: people demand about a $30 \%$ reduction in the premium to compensate them for a $1 \%$ chance that their claim will not be paid. This finding agrees with Merton's introspection, as well as with the results of similar surveys summarized below. Indeed, it is not surprising that risk averse decision makers would require substantially more than a $1 \%$ reduction in the premium to compensate for a $1 \%$ risk of not being paid. What is surprising, however, is that no substantial reduction beyond $1 \%$ can be accommodated by expected utility theory with any plausible utility function. To illustrate, assume a logarithmic utility function $\ln (x+k)$. According to this function, if the reservation price (i.e., the most the decision maker is willing to pay) in question (A1) is $\$ 700$ (implying $k=602,980$ ) then the reservation price for probabilistic insurance should be $\$ 692.9952$, i.e., about half a cent less than the 
actuarial $\$ 7.00(1 \%)$ reduction in the premium; a far cry from the median response of $\$ 500$ (a 28\% reduction). As will be shown later, similar results hold for other utility functions.

It is noteworthy that, while the majority of the respondents were willing to pay above the actuarially fair premium for standard insurance (Questions 1 and 3), the majority of respondents were not willing to pay the actuarially fair premium for probabilistic insurance (Questions 2 and 4). Note also that doubling the replacement cost for the house has little effect on the ratio of the reservation prices for a standard and probabilistic insurance. As will be shown later, these observations are consistent with prospect theory.

We have studied attitudes regarding probabilistic insurance in other contexts using two other groups of respondents. These surveys differed from survey 1, described above, in that we did not elicit a reservation price for standard insurance. Instead, we specified this price and asked the respondents how much less they would pay for the probabilistic version with a $1 \%$ default risk. The results of these surveys are given in Table 1 which also summarizes the data from survey 1 .

In surveys 2 and 3 the respondents were also asked to state their reservation price for standard insurance assuming that the amounts at risk were increased by $15 \%$ or $10 \%$, respectively. In both cases, the median prices increased proportionally, as implied by constant relative risk aversion. Although survey data of this type have obvious limitations, they clearly suggest that people find the concept of probabilistic insurance unattractive.

\section{Expected utility}

We analyze here the simplest examples of standard and probabilistic insurance. We assume that with no insurance (NI) one loses $Z$ if the hazard $H$ occurs, and nothing if it does not (see Figure 1a). ${ }^{1}$ Standard insurance (SI) is illustrated in Figure 1b. Here one pays a premium $y$, and is fully reimbursed if the hazard occurs. Probabilistic insurance (PI) is illustrated in Figure 1c. Here one pays a reduced premium, $x$, but if the hazard $H$ occurs, there is a possibility $R$ that the claim will not be reimbursed. ${ }^{2}$ We assume that the premiums $y$ and $x$ are the reservation prices for SI and PI, respectively, so that the decision maker is indifferent between SI and NI for premium $y$, and between PI and NI for

Table 1. Summary of probabilistic insurance surveys.

\begin{tabular}{lllll}
\hline Survey Respondents & Context & $y$ & $\mathrm{x}$ & $\mathrm{x} / \mathrm{y}$ \\
\hline 1. University students & fire insurance & $\$ 700$ & $\$ 500$ & 0.71 \\
$\mathrm{~N}=86$ & fire insurance & $\$ 1300$ & $\$ 900$ & 0.69 \\
2. University students & car insurance & $\$ 100$ & $\$ 70$ & 0.70 \\
$\mathrm{~N}=144$ & & & \\
3. Money managers & international investment & $\$ 1000$ & $\$ 750$ & 0.75 \\
$\mathrm{~N}=75$ & & & \\
\hline
\end{tabular}

$\mathrm{y}$ : reservation price for standard insurance

$\mathrm{x}$ : elicited reservation price for a $1 \%$ probabilistic insurance 

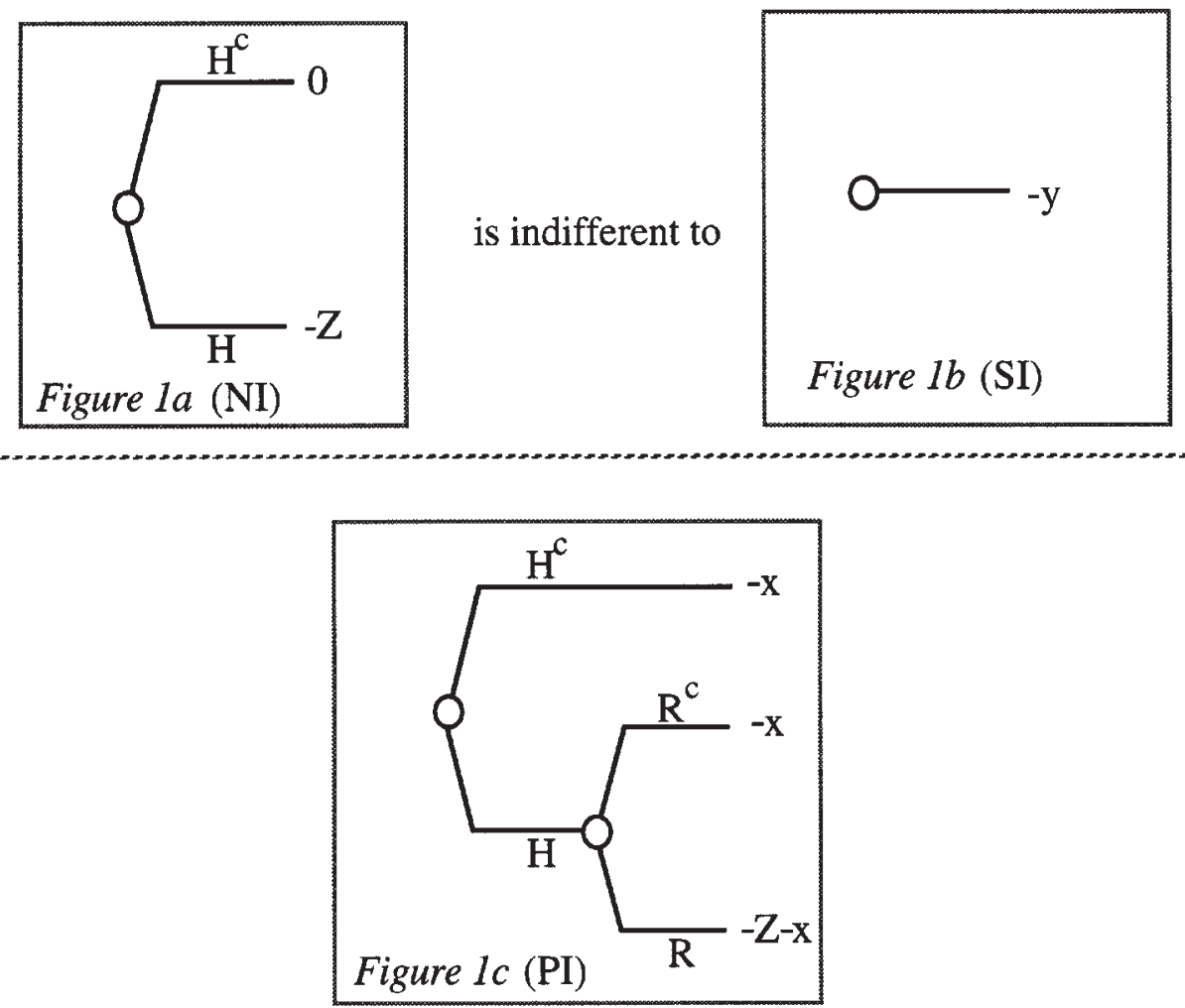

Figure 1. (Question: Is PI preferred to any of the upper two?).

premium $x$. We study the relation between $x$ and $y$. Naturally, the greater the reluctance to purchase PI, the lower the reservation price. Implications for the case where the premiums do not coincide with the reservation prices are discussed later.

This section assumes expected utility theory. However, the analysis is readily extended to nonexpected utility models based on decision weights. For this reason, we denote the utility function by $v$, and set $v(0)=0$. Throughout the paper we assume that $v$ has a positive continuous derivative $v^{\prime}$. Let $p$ be the probability of the event $H ; p$ can be either an objective or a subjective probability. According to expected utility theory, if the decision maker is indifferent between SI and NI, then

$$
p v(-Z)=v(-y) .
$$

The expected utility of PI is

$$
\operatorname{prv}(-Z-x)+(1-p r) v(-x)
$$


where $r$ is the conditional probability of $R$ given $H$, i.e. the probability that the insurance company will not pay given that the hazard has occurred.

Recall that in PI, the insurance company reimburses the consumer only in a $(1-r)$-fraction of the cases. Hence $(1-r) y$ is called the actuarially adjusted premium for PI. This would be the premium for PI assuming linear utility, and it provides a useful standard of comparison for $x$. The results of our survey, however, indicate that $x$ was considerably lower than $(1-r) y$, presumably because people did not like the additional risk entailed by PI. The following theorem provides limits for $x$ under expected utility theory with a concave, i.e. risk averse, utility function. The expressions are somewhat complex, but are discussed after. A simpler first-order approximation is provided subsequently, in Theorem 2.2.

Theorem 2.1 (Upper and lower bounds for $x$ ). If $v$ is concave then

$$
(1-r) y+r y\left(1-\frac{v^{\prime}(0)}{v^{\prime}(-y)}\right) \geq \mathrm{x} \geq(1-r) y-r y p\left(\frac{v^{\prime}(-Z-y)}{v^{\prime}(0)}-1\right) . \square
$$

Note that, by concavity of $v, v^{\prime}(0) / v^{\prime}(-y)$ is less than 1 , hence the upper bound is greater than $(1-r) y$. Similarly, $v^{\prime}(-Z-y) / v^{\prime}(0)$ is greater than 1 , hence the lower bound is less than $(1-r) y$. However, as we next show informally, in a first-order approximation the upper and lower bound are essentially equal to $(1-r) y$, which is the actuarially adjusted premium. A formal derivation is presented in the Appendix.

Consider the plausible assumption that $v$ is approximately linear on the interval $[-y, 0]$ and $v^{\prime}(0)$ is approximately equal to $v^{\prime}(-y)$. Hence the upper bound for $x$ is approximately $(1-r) y$. Turning to the lower bound, note that $v^{\prime}(-Z-y)$ is larger than $v^{\prime}(0)$ by concavity. Our approximation is based on the assumption that $v^{\prime}(-Z-y) / v^{\prime}(0)$ may be large, say 10 , but is not extreme and is considerably smaller than $1 / p$, which typically exceeds 100. These conditions are likely to be met if the loss of $Z$ is not ruinous. All our survey questions appear to meet this condition. Under the above assumptions, the second term of the lower bound,

$$
-r y p\left(\frac{v^{\prime}(-Z-y)}{v^{\prime}(0)}-1\right)
$$

is much smaller than $r y$. In other words, the lower bound for $x$, like the upper bound, is also approximately $(1-r) y$. As we prove in the appendix, this approximation does not depend on the concavity of $v$.

Theorem 2.2. The reservation price $x$ for PI is approximately ${ }^{3}$ equal to the actuarially adjusted premium $(1-r) y$.

This result is quite surprising. It shows that in a first-order approximation, the reservation price for PI according to expected utility theory is very close to the actuarially adjusted premium. In other words, the curvature of utility has essentially no impact. 
Whether the reservation price $x$ is actually higher or lower than the actuarially adjusted premium for concave utility, depends on second-order effects. An examination of the proof of Theorem 2.1 reveals another surprising fact: For some concave utility functions, $x$ is in fact higher than the actuarially adjusted premium $(1-r) y$. This is proved in the Appendix (Proposition A.2; see also the elucidation above that proposition), and illustrated in Table 2 (Examples 9, 10, and 11). Hence, a decision maker with a concave utility may prefer actuarially adjusted PI to SI although PI appears "riskier" than SI.

Table 2 presents numerical results for PI for twenty concave utility functions, described in the first column. ${ }^{4}$ All the functions belong to the HARA-family. This family was defined by Merton (1971) and contains the commonly used parametric forms. The utility functions in Table 2 form five groups with four functions in each group. The first group (1-4) includes logarithmic functions. The second group (5-8) includes positive powers of positive numbers. The third group (9-12) includes power functions on the negative reals. Here, the exponent exceeds one to ensure concavity, and the values raised to the given power are positive (zero is excluded by the requirement that $v^{\prime}>0$ ). The fourth group (13-16) includes hyperbolic functions, and the last group (17-20) includes exponential functions. For each family an example is considered with an extreme degree of global risk aversion $\left(v^{\prime}(-Z) / v^{\prime}(0)=100\right.$ or 1000), an example with substantial global risk aversion $\left(v^{\prime}(-Z) / v^{\prime}(0)=25\right)$, and two examples with small global risk aversion $\left(v^{\prime}(-Z) / v^{\prime}(0)=\right.$ 2).

Table 2. Summary of numerical results for a $1 \%$ probabilistic insurance for twenty concave utility functions.

\begin{tabular}{|c|c|c|c|c|c|c|}
\hline UTILITY & $\frac{v^{\prime}(-Z)}{v^{\prime}(-0)}$ & $\frac{v^{\prime}(-y)}{v^{\prime}(-0)}$ & $p$ & $\mathrm{y}$ & $\mathrm{Z}$ & $\frac{x}{y}$ \\
\hline $1 \ln (z+100,100.1)$ & 1000.0 & 1.00007 & 0.00001 & 6.914 & 100,000 & 0.98990 \\
\hline $2 \ln (z+104,166)$ & 25.0 & 1.003 & 0.001 & 334.774 & 100,000 & 0.98977 \\
\hline $3 \ln (z+200,000)$ & 2.0 & 1.001 & 0.001 & 138.581 & 100,000 & 0.989994 \\
\hline $4 \ln (z+200,000)$ & 2.0 & 1.003 & 0.005 & 691.947 & 100,000 & 0.98997 \\
\hline $5(z+100,500)^{0.1316}$ & 100.0 & 1.001 & 0.00026 & 100 & 100,000 & 0.98972 \\
\hline $6(z+100,500)^{0.393}$ & 25.0 & 1.001 & 0.00044674 & 100 & 100,000 & 0.98989 \\
\hline $7(z+100,300)^{0.88}$ & 2.0 & 1.0001 & 0.00088 & 100 & 100,000 & 0.989991 \\
\hline $8(z+150,000)^{0.3691}$ & 2.0 & 1.004 & 0.0074 & 1000 & 100,000 & 0.98995 \\
\hline $9-(-z+1)^{1.6}$ & 1000.0 & 63.1 & 0.00063 & 1000 & 100,000 & 0.9936 \\
\hline $10-(-z+1)^{1.28}$ & 25.0 & 6.9 & 0.00276 & 1000 & 100,000 & 0.992 \\
\hline $11-(-z+1)^{1.0605}$ & 2.0 & 1.5 & 0.00757 & 1000 & 100,000 & 0.9905 \\
\hline $12-(-z+100,000)^{2}$ & 2.0 & 1.01 & 0.0067 & 1000 & 100,000 & 0.98998 \\
\hline $13(-z+27,010)^{-6}$ & 100.0 & 1.3 & 0.005 & 1000 & 13020.7 & 0.986 \\
\hline $14(-z+54,500)^{-2}$ & 25.0 & 1.06 & 0.005 & 1000 & 35857.6 & 0.9890 \\
\hline $15(-z+385,000)^{-0.5}$ & 2.0 & 1.004 & 0.005 & 1000 & 142590.8 & 0.98997 \\
\hline $16(-z+485,000)^{-1}$ & 2.0 & 1.004 & 0.005 & 1000 & 141812.9 & 0.98997 \\
\hline $171-\exp (-0.000692645 z)$ & 1000.0 & 2.0 & 0.001 & 1000 & 9973 & 0.986 \\
\hline $181-\exp (-0.00113329 z)$ & 25.0 & 1.1 & 0.005 & 100 & 2840.3 & 0.9894 \\
\hline $191-\exp (-0.000001 z)$ & 2.0 & 1.001 & 0.001 & 1000 & 693397.2 & 0.989995 \\
\hline $201-\exp (-0.00005 z)$ & 2.0 & 1.005 & 0.005 & 100 & 13888 & 0.98998 \\
\hline
\end{tabular}


The last column in Table 2 gives the reservation price $x$ for PI, divided by $y$. The lowest value of the quotient $x / y$ in the table is 0.986 , which is close to the actuarially adjusted premium of 0.99 , and very far from the values observed in our surveys. As noted above, in some cases $(9,10$, and 11$)$ the ratio exceeds 0.99 , showing that a concave utility function can give rise to a preference for probabilistic insurance. These results illustrate the preceding analysis and show that the pronounced aversion to probabilistic insurance cannot be explained by any realistic curvature of utility.

The preceding analysis has demonstrated that, under expected utility, the reservation price for PI approximately coincides with the actuarial adjustment of the reservation price for SI. This holds regardless of whether the actual premium coincides with the reservation price. In reality, of course, the premium set by the insurance company may fall either above or below a consumer's reservation price. We next consider these possibilities. Given a noncompulsary standard insurance, we distinguish among three classes of consumers: the insured, whose reservation price exceeds the actual premium; the uninsured, whose reservation price is below the premium; and the indifferent, whose reservation price coincides with the premium. We assume that expected utility holds and that PI, with an actuarially adjusted premium, is made available.

First we suppose that SI is no longer available, i.e., that PI has replaced SI. In this case, people who bought SI will now buy PI, and people who did not buy SI will not buy PI. To verify, recall that the reservation price for PI is approximately $(1-r)$ times the reservation price for SI, and the premium for PI is $(1-r)$ times the premium for SI. Therefore, the reservation price for PI exceeds the PI premium if and only if the reservation price for SI exceeds the SI premium. In other words, replacing SI by an actuarially adjusted PI will not change the status of the insured and the uninsured. Expected utility, therefore, predicts that the insurance industry would not lose many clients if SI were replaced by an actuarially adjusted PI. Only clients whose reservation price for SI is very close to the actual premium might be lost.

Second, let us also consider the case where SI remains available, so that the option of PI is added to the option of SI, and let us see what expected utility predicts here. In this case, consumers are unlikely to purchase PI. For the consumers who have a strong preference for SI over NI, PI is not very attractive because it involves an additional risk. For the consumers who have a strong preference for NI over SI, actuarially adjusted PI is not an attractive option because the premium remains too close to the SI premium, which was deemed too high. For consumers who are roughly indifferent between SI and NI, Theorem 2.2 applies, and they will be approximately indifferent between these options and PI.

In summary, expected utility makes the counterintuitive prediction that not many clients will be lost if SI is replaced by PI at actuarially adjusted premiums, and new clients will be attracted if the PI premium is actuarially favorable. We conclude this section with a numerical example of the case where the actual premium is lower than the reservation price, so that SI yields consumer surplus. Suppose that SI is offered at an actuarially fair premium of $\$ 400$, and that $Z=\$ 100,000$, thus $p=0.004$, and $r=0.01$. Assume a risk averse utility function $v(z)=\ln (300,000-z)$, so that the consumer indeed prefers SI over NI. According to expected utility, the premium that makes probabilistic insurance as attractive as SI in this case is $\$ 395.12$. It is noteworthy that this value is less than, but very 
close to, the actuarially adjusted premium of $\$ 396$, even though the SI premium here is well below the reservation price.

\section{Prospect theory}

\subsection{Basic results}

We next show that reluctance to purchase probabilistic insurance can be explained by the weighting function of prospect theory. ${ }^{5}$ Because all outcomes are nonpositive, prospect theory coincides with the rank-dependent theories for risk (Quiggin, 1981) and uncertainty (Schmeidler, 1989). Again, we assume in this section that $y$ and $x$ are the reservation prices for SI and PI, respectively. According to prospect theory, the overall value of SI is $v(-y)$ and the value of NI is $p v(-Z)$, where $v$ is the value function, $p=W^{-}(H)$, and $W^{-}$ is the weighting function for losses. Since we consider only nonpositive outcomes, the superscript is suppressed. Again it is assumed that $v^{\prime}$ is positive and continuous.

The value of PI (see Figure 1c) now is

$$
\operatorname{prv}(-Z-x)+(1-p r) v(-x)
$$

where $r$ is defined below. There are two different ways of applying prospect theory to the two-stage tree in Figure 1c: the reduced form and the stepwise form. In the reduced form (sometimes called the normal form), the value of PI is

$$
W(H R) v(-Z-x)+(1-W(H R)) v(-x)
$$

where $W(H R)$ is the decision weight associated with the conjunction of the events $H$ in the first stage and $R$ in the second. The decision weight of $v(-Z-x)$ can be expressed as $p r$ by defining

$$
r=\frac{W(H R)}{p}=\frac{W(H R)}{W(H)},
$$

which in general is different from $W(R)$.

The stepwise form (sometimes called backward induction) proceeds as follows. First, one evaluates the $H$-branch of the tree as

$$
W(R) v(-Z-x)+(1-W(R)) v(-x) .
$$

Second, the value of PI is obtained as

$$
p(W(R) v(-Z-x)+(1-W(R)) v(-x))+(1-p) v(-x),
$$

which can be rewritten as 


$$
p W(R) v(-Z-x)+(1-p W(R)) v(-x) .
$$

By setting $r=W(R)$, the above equation reduces to Equation (3.1).

Because SI, NI, and PI are all indifferent by assumption, we obtain

$$
p v(-Z)=v(-y)=\operatorname{prv}(-Z-x)+(1-p r) v(-x) .
$$

Note that the same relations hold for expected utility except that now the weights $p$ and $r$ have a different interpretation. Therefore Theorems 2.1 and 2.2 hold with the new interpretation.

Theorem 3.1. If $v$ is concave, then

$$
(1-r) y+r y\left(1-\frac{v^{\prime}(0)}{v^{\prime}(-y)}\right) \geq x \geq(1-r) y-r y p\left(\frac{v^{\prime}(-Z-y)}{v^{\prime}(0)}-1\right) . \square
$$

Theorem 3.2. The reservation price $x$ is approximately $(1-r) y$. $\square$

Under the present interpretation, $r$ is defined in terms of (subjective) decision weights. Consequently, $(1-r) y$ is no longer the actuarially adjusted premium. Suppose that we are given an objective probability of $R$ conditional on $H$, denoted by $r^{*}$. Obviously, $r^{*}$ may differ from the decision weight $r$. We can rewrite $(1-r) y$, the first-order approximation for $x$, as

$$
x=\left(1-r^{*}\right) y-\left(r-r^{*}\right) y .
$$

That is, $x$ can be expressed as the actuarially adjusted premium $\left(1-r^{*}\right) y$ minus a component that reflects the discrepancy between the decision weight $r$ and the objective probability $r^{*}$. Letting $X$ denote the premium to be paid for PI, preference for PI over NI means

$$
X \leq x=\left(1-r^{*}\right) y-\left(r-r^{*}\right) y,
$$

or

$$
\left(1-r^{*}\right) y-X \geq\left(r-r^{*}\right) y \text {. }
$$

The left-hand side is the "actuarial gain" for the decision maker and the right-hand side reflects the deviation of decision weight from objective probability. Thus PI is acceptable if and only if the left-hand side (the actuarial gain) exceeds the right-hand side (the deviation from expected utility). In accord with experimental data, prospect theory assumes that decision weights are subadditive (i.e., the most extreme outcomes are overweighted and intermediate outcomes are underweighted; see Tversky \& Kahneman, 1992; 
Camerer \& Ho, 1994; Wu \& Gonzalez, 1995; Tversky \& Wakker, 1995). Hence $r$ will be substantially greater than $r^{*}$ for unlikely events, which explains why PI is highly unattractive. A numerical example is given in Subsection 3.4 below.

The above discussion may be summarized as follows:

(a) In a first-order approximation, PI is acceptable if and only if the actuarial gain exceeds the deviation from expected utility.

(b) The value function is much less important than the weighting function for explaining the aversion to PI.

\subsection{Partial insurance}

It is important to distinguish between the concept of probabilistic insurance addressed in this paper and the concept of partial insurance. In partial insurance, the consumer pays a fraction $(1-r) y, 0<r<1$, of the regular premium $y$, and in return is reimbursed only for a fraction $(1-r) Z$ of the damage if the hazard occurs. Thus, partial insurance is essentially equivalent to insurance with a deductable or co-insurance feature. Note that in both partial and probabilistic insurance the consumer pays a reduced premium and receives less than full coverage; but while probabilistic insurance yields either full coverage or no coverage at all, thereby exposing the consumer to additional risk if the hazard occurs, partial insurance always pays a fixed portion of the damage. As noted by some authors (see, e.g., Mossin, 1968; Borch, 1974), consumers' degree of dislike for deductables is at variance with the predictions of expected utility theory and risk aversion. Although consumers appear to undervalue both partial and probabilistic insurance, relative to the prediction of the standard theory, the departures from the theory appear more pronounced in the latter than in the former. As an illustration of this claim, we conducted a survey of $57 \mathrm{MBA}$ students at the University of Chicago. Each student was asked to express a willingness to pay for three different fire insurance policies on a $\$ 125,000$ house with no mortgage. They were told that the risk of a fire was 1 in 200 . The three policies they considered were standard insurance, .99 probabilistic insurance, and .99 partial insurance (99 percent of any claim will be paid). For each subject we computed the ratio of the willingness to pay for the probabilistic and partial insurance to the standard insurance. For probabilistic insurance the median ratio was .50, but for partial insurance it was much higher, .95 (the difference is highly significant). For a discussion of partial insurance from the standpoint of rank-dependent utility theory, essentially using its nondifferentiability, see Segal \& Spivak (1990).

\subsection{Testing assumptions}

The preceding analysis is based on prospect theory. It uses two assumptions (see the discussion following Theorem 2.1): 
(a) that $v^{\prime}$ is not extremely greater around $-Z$ than around 0 ;

(b) that $v$ is approximately linear on the small interval $[-y, 0]$.

Because (b) is noncontroversial, we focus on the testing of assumption (a).

Assume again that NI and SI are indifferent. Suppose that the values $p^{*}$ (probability of $H$ ) and $Z$ are explicitly given to the subject. Then the reservation price $y$ for SI is given by the equation

$$
p v(-Z)=v(-y)
$$

Next ask the subject how much more than $y$ she would be willing to pay if the damage $Z$ were larger by an amount $a>0$. The reservation price $y+b(b>0)$ is elicited such that

$$
p v(-Z-a)=v(-y-b) .
$$

This procedure can be used for testing relative risk aversion. Under expected utility with constant relative risk aversion (for negative amounts), $a / Z=b / y$. It turns out that this procedure can also be used to test that the quotient $v^{\prime}(-Z) / v^{\prime}(0)$ is not too extreme. Roughly, if $y$ is relatively small in comparison to $Z$ and $v^{\prime}(-Z)$ is much greater than $v^{\prime}(0)$, then there may be a discrepancy between the reservation price $x$ and $(1-r) y$, but it does not exceed $(b / a) r y$. Precise results are presented in the Appendix.

Theorem 3.3. If $v$ is concave, then in a first-order approximation,

$$
x \geq(1-r) y-\left(\frac{b}{a}\right) r y \cdot \square
$$

Thus, $v^{\prime}(-Z)$ departs from $v^{\prime}(0)$ so much that $x$ differs substantially from $(1-r) y$ only if $b / a$ is nonnegligible. This property is tested in surveys 1,2 , and 3 (see Table 1). The given value of $a$, the increase in the damage $Z$, was $100 \%, 15 \%$, and $10 \%$, respectively. The median increments in the reservation prices were exactly proportional in each case. Under expected utility, the reservation price $x$ for PI should then not depart from the actuarially adjusted premium by more than $(b / a)$ ry $=(\mathrm{y} / \mathrm{Z}) r y$, which is considerably smaller than the observed departure of nearly $30 \%$. These observations confirm our assumptions regarding $v^{\prime}(-Z)$.

Our survey data can also be used to test a prediction of prospect theory, assuming again that $v$ is not extremely curved on $[-y, 0]$. According to prospect theory, the reluctance to purchase PI is driven by the weighting function, not by the value function. Consequently, prospect theory implies that the ratio $x / y$ should remain roughly the same if the probabilities are fixed and the payments are varied as follows. Assume again that the subject is indifferent between NI and SI, and that $x$ is the reservation price for PI. Now the subject is asked to consider the case where, for the same probability $p^{*}$ of the event $H$, the damage $Z^{\prime}$ is considerably different from $Z$, say $Z^{\prime}=2 Z$. Then the new reservation price $y^{\prime}$ for SI will also be considerably different from $y$ (under expected value maximization, $y^{\prime}=2 y$ ). 
The subject is next asked what is her reservation price $x^{\prime}$ for PI. Prospect theory predicts that $x^{\prime} / y^{\prime}$ is again close to $1-r$, hence $x^{\prime} / y^{\prime}$ should be approximately equal to $x / y$. Indeed, the ratios of the median values in survey 1 are $x / y=0.71$ and $x^{\prime} / y^{\prime}=0.70$.

\subsection{The weighting function}

We now show that the aversion to PI is consistent with the general characteristics of risky weighting functions, such as subproportionality (i.e., $w\left(q p^{*}\right) / w\left(p^{*}\right)$ decreases with $p^{*}$, see Kahneman \& Tversky, 1979). To illustrate, consider the following parametric family, introduced and characterized by Prelec (1995).

$$
w(p)=\exp \left(-\beta(-\ln p)^{\alpha}\right)
$$

We have chosen this family because it satisfies subproportionality for the small probabilities that are relevant to our analysis. Prelec suggested the parameter values $\alpha=0.65$ and $\beta=1$ because they agree with the data of Tversky \& Kahneman (1992) and others.

Suppose that the probability $r^{*}$ of no payment given the event $H$ is 0.01 . Assuming the reduced form for evaluating PI, the decision weight of the event $R$ in the second stage (see Figure 1c) is $w\left(0.01 p^{*}\right) / w\left(p^{*}\right)$ where $p^{*}$ is the probability of $H$. The reservation price $x$ for probabilistic insurance is the same as the actuarially adjusted premium if $r$ (instead of $r^{*}$ ) were the conditional probability of $R$ given $H$. That is, then $x$ is $(1-r) y$. Substituting (3.2) yields $x=0.773 y$ for $p^{*}=0.005$ and $x=0.749 y$ for $p^{*}=0.001$, which are in general agreement with our survey data.

Two comments regarding this analysis are in order. First, we have assumed a linear value function so that probabilistic insurance is explained solely by the curvature of the weighting function. A linear value function for losses, of course, is a limiting case of the concavity assumption commonly invoked in expected utility theory, and of the convexity assumption suggested by prospect theory. ${ }^{6}$ Second, the preceding evaluation of PI has been based on the reduced form, yielding $w\left(0.01 p^{*}\right) / w\left(p^{*}\right)=r=0.25$, which appears reasonable. In contrast, the stepwise evaluation of the two-stage PI tree yields $w(0.01)=$ $r=0.07$, which appears too low. The reduced form seems to provide a more adequate account of PI than the stepwise evaluation, although in other contexts the latter has been favored by several authors (Loomes \& Sugden, 1986; Chew \& Epstein, 1989; Segal, 1990; Luce \& von Winterfeldt, 1994).

\section{Discussion}

The main results of this paper may be summarized as follows. First, we have observed that people dislike probabilistic insurance: Most respondents demanded more than $20 \%$ reduction in premium to offset a $1 \%$ default risk. Second, we have demonstrated that such preferences are generally inconsistent with expected utility theory. Under highly plausible 
assumptions about utility for money, the reduction in premium should be essentially equal to the default risk. Third, we have shown that the reluctance to buy probabilistic insurance is predicted by the weighting function of prospect theory.

In the classical economic analysis, insurance is explained by concavity of utility. In prospect theory, insurance is explained by subadditivity of decision weights. Probabilistic insurance offers a critical comparison of the two accounts. The observed aversion to probabilistic insurance suggests that the purchase of insurance is driven primarily by the overweighting of small probabilities rather than by diminishing marginal utility in prospect theory (see also Viscusi, 1995). An adequate analysis of insurance, therefore, should attend to nonlinearity of chance, not merely to the nonlinearity of value.

To illustrate the point, consider a situation in which the cost of reducing the default risk $(r)$ to zero or near zero (e.g., going from AA to AAA credit rating, or providing complete coverage for war damages) is very high. In this case, firms can afford to offer probabilistic insurance at rates that are significantly lower than the actuarially adjusted standard insurance. A utility-based analysis predicts that such offers will attract many clients, whereas an analysis based on decision weights predicts that they will not.

In the terms of prospect theory, the reluctance to purchase probabilistic insurance is another manifestation of the certainty effect, which underlies Allais' famous example. Although the two problems appear quite different (Allais' example involves substantial gains, whereas probabilistic insurance involves substantial losses), in both cases a $1 \%$ move away from certainty produces a dramatic reduction in the attractiveness of a prospect.

The reluctance to purchase insurance policy with an explicit default risk is particularly striking in light of the fact that essentially all insurance policies are probabilistic, even though they are rarely described in such terms. The marketing of insurance, which typically does not mention the default risk, is designed to create what amounts to pseudocertainty (Kahneman \& Tversky, 1979). Once the default risk is made explicit, however, it greatly reduces the attractiveness of the policy.

In everyday life, people usually discuss risk in verbal qualitative terms that are not readily translatable into the probability language. For example, a bond with an AAA rating is considered to be essentially risk-free, and a formula approved for infants is meant to be riskless, although certainty cannot be achieved in either case. In these contexts, a drop below these standards may be perceived as a dramatic change in risk. If buyers of securities treat an AAA rating as risk free, and anything else as risky, then, as noted by Merton (1993), issuers must go to great lengths to achieve the illusion of zero risk.

Although probabilistic insurance seems somewhat unusual, it is a prototype of most forms of protective action in which one invests some resources to reduce the probability of some hazard, without eliminating it altogether. Examples are medical check-ups, the installation of burglar alarm, and the purchase of new tires. In all these cases, the consumer's action reduces but does not eliminate the probability of the hazard in question. The finding that people value the elimination of risk disproportionally more than the reduction of risk represents a major departure of human behavior from the canons of rational choice. 


\section{Appendix. Proofs}

Throughout the appendix we assume that the value of SI is $v(-y)$, the value of NI is $p v(-Z)$, the value of PI is $p r v(-Z-x)+(1-p r) v(-x)$, and that these values are the same:

$$
\mathrm{v}(-\mathrm{y})=\operatorname{pv}(-\mathrm{Z})=\operatorname{prv}(-\mathrm{Z}-\mathrm{x})+(1-\operatorname{pr}) \mathrm{v}(-\mathrm{x})
$$

We first introduce a notation that simplifies the approximations in which derivatives of $v$ are used. For the value function $v$ and arguments $a>b$, let $m(b, a)$ denote the average derivative of $v$ over the interval $[b, a]$, i.e.

$$
m(b, a)=\frac{v(a)-v(b)}{a-b} .
$$

For a concave utility $v, v^{\prime}(b) \geq m(b, a) \geq v^{\prime}(a)$, and $m(b, a)$ decreases in $a$ and $b$, i.e. it is smaller as the interval is "more to the right."

Whenever we substitute a derivative $v^{\prime}(a)$ or $v^{\prime}(b)$ for $m(a, b)$ below, we ensure that $a$ is close to $b$, so that the substitution does not generate a large distortion. As a preparation for the proofs of Theorems 2.1 and 2.2, we show:

Lemma A.1 (Exact implicit equation for $x$ ).

$$
x=(1-r) y+\frac{r x(m(-y,-x)-m(-x, 0))-\operatorname{rxp}(m(-Z-x,-Z)-m(-x, 0))}{m(-y,-x)} .
$$

Proof. The equalities in (A.1) imply that the third term minus $r$ times the second term minus $1-r$ times the first term is zero, i.e.

$$
\begin{gathered}
0=p r v(-Z-x)+(1-p r) v(-x)-r p v(-Z)-(1-r) v(-y)= \\
-p r(v(-Z)-v(-Z-x)+v(-x))+v(-x)-(1-r) v(-y)= \\
-p r(v(-Z)-v(-Z-x)-(v(0)-v(-x)))+(1-r)(v(-x)-v(-y))-r(v(0) \\
-v(-x))
\end{gathered}
$$

Note that all value differences in the formula are over intervals of length $x$ or $y-x$, i.e. intervals of length of smaller order of magnitude than $Z$. To see that in a first-order approximation, expected utility is close to expected value maximization, note that the first part of the formula is preceded by a factor $p$ which is small; hence this first part can be ignored in a first-order approximation (for $y$ and thus $p$ tending to 0 and the $v$-values fixed). The second part of the formula only concerns outcomes from the interval $[-y, 0]$, 
and for such moderate amounts expected utility is close to expected value. An exact analysis of these points is provided in the proof of Theorem 2.2. Continuing the proof of Lemma A.1, the last formula is equal to:

$$
\begin{gathered}
-\operatorname{rxp}(m(-Z-x,-Z)-m(-x, 0))+(1-r)(y-x) m(-y,-x)-\operatorname{rxm}(-x, 0)= \\
-\operatorname{rxp}(m(-Z-x,-Z)-m(-x, 0))+(1-r) y m(-y,-x)-(1-r) x m(-y,-x) \\
-r x m(-x, 0)= \\
-r x p(m(-Z-x,-Z)-m(-x, 0))+(1-r) y m(-y,-x)-x m(-y,-x)+ \\
r x(m(-y,-x)-m(-x, 0))
\end{gathered}
$$

which implies that

$$
\begin{aligned}
r x(m(-y,-x)-m(-x, 0)) & -\operatorname{rxp}(m(-Z-x,-Z)-m(-x, 0))= \\
& +m(-y,-x)(x-(1-r) y) .
\end{aligned}
$$

Consequently,

$$
x-(1-r) y=\frac{r x(m(-y,-x)-m(-x, 0))-\operatorname{rxp}(m(-Z-x,-Z)-m(-x, 0))}{m(-y,-x)},
$$

which implies the equality in the lemma.

Proof of theorem 2.1. We first derive the last inequality, concerning the lower bound, starting from the equality in Lemma A.1.

$$
\begin{gathered}
x-(1-r) y=\frac{\operatorname{rx}(m(-y,-x)-m(-x, 0))-\operatorname{rxp}(m(-Z-x,-Z)-m(-x, 0))}{m(-y,-x)} \\
\geq-\frac{\operatorname{rxp}(m(-Z-x,-Z)-m(-x, 0))}{m(-y,-x)} \geq-\frac{\operatorname{rxp}\left(v^{\prime}(-Z-x)-v^{\prime}(0)\right)}{v^{\prime}(x)} \\
\geq-\frac{\operatorname{ryp}\left(v^{\prime}(-Z-y)-v^{\prime}(0)\right)}{v^{\prime}(0)}=-\operatorname{ryp}\left(\frac{v^{\prime}(-Z-y)}{v^{\prime}(0)}-1\right)
\end{gathered}
$$

from which the lower bound in the theorem follows.

Next we turn to the derivation of the upper bound. By Lemma A.1,

$$
\begin{gathered}
x-(1-r) y=\frac{r x(m(-y,-x)-m(-x, 0))-\operatorname{rxp}(m(-Z-x,-Z)-m(-x, 0))}{m(-y,-x)}= \\
r x-\frac{r x p m(-Z-x,-Z)+m(-x, 0)(r x-r x p)}{m(-y,-x)} \leq
\end{gathered}
$$




$$
\begin{gathered}
r x-\frac{r x p v^{\prime}(-Z)+v^{\prime}(0)(r x-r x p)}{v^{\prime}(-y)}= \\
r x-\frac{r x p\left(v^{\prime}(-Z)-v^{\prime}(0)\right)+v^{\prime}(0) r x}{v^{\prime}(-y)}= \\
r x\left(\frac{v^{\prime}(-y)-v^{\prime}(0)-p\left(v^{\prime}(-Z)-v^{\prime}(0)\right)}{v^{\prime}(-y)}\right) \leq \\
r x\left(\frac{v^{\prime}(-y)-v^{\prime}(0)}{v^{\prime}(-y)}\right) \leq r y\left(\frac{v^{\prime}(-y)-v^{\prime}(0)}{v^{\prime}(-y)}\right)=r y\left(1-\frac{v^{\prime}(0)}{v^{\prime}(-y)}\right)
\end{gathered}
$$

from which the upper bound in the theorem follows.

If $v$ is strictly concave in the neighborhood of 0 , but is linear or only weakly concave for more negative arguments, then the reservation price $x$ can be larger than the actuarially adjusted premium (see Examples 9, 10, and 11 in Table 2). More generally, we have the following result.

Proposition A.2. Assume that $v$ is concave, $v^{\prime}(0)<v^{\prime}(-x)$, and $v^{\prime}(-Z-x)=v^{\prime}(-x)$ so that $v$ is linear on the interval $[-Z-x,-x]$. Then $x>(1-r) y$.

Proof. Consider the equality in Lemma A.1. We can rewrite it as

$$
\begin{array}{r}
x=(1-r) y+\frac{r x\left(v^{\prime}(-y)-m(-x, 0)\right)-r x p\left(v^{\prime}(-y)-m(-x, 0)\right)}{v^{\prime}(-y)} \\
=(1-r) y+\frac{r x(1-p)\left(v^{\prime}(-y)-m(-x, 0)\right)}{v^{\prime}(-y)} .
\end{array}
$$

By continuous differentiability, $v^{\prime}(0)<v^{\prime}(-x)$ implies $m(-x, 0)<v^{\prime}(-x)=v^{\prime}(-y)$, therefore the quotient is positive and $x>(1-r) y$.

Proof of theorem 2.2. Note that concavity of $v$ is not assumed in this proof. We derive a first-order approximation for $y \downarrow 0$, i.e. for $y$ tending to 0 from above. Here $r$ and $Z$ are kept fixed. $x$ also tends to 0 , as $-y<-x<0$, and so does $p$ because $p=v(-y) / v(-Z){ }^{7}$ We write $o(z)$ for any function $f(z)$ such that $\lim _{z \downarrow 0} f(z) / z=0$. The actuarially fair adjustment of the premium when SI is changed into PI requires a reduction by $r y$, therefore $r y$ seems a relevant order of magnitude. Given that $r$ is a constant, $o(r y)$ formally agrees with $o(y)$, and therefore our formal results are stated in terms of $o(y)$. Thus, a formal statement of Theorem 2.2 is:

$$
x-(1-r) y=o(y)
$$


This is demonstrated next. By Lemma A.1,

$$
x-(1-r) y=\frac{r x(m(-y,-x)-m(-x, 0))-\operatorname{rxp}(m(-Z-x,-Z)-m(-x, 0))}{m(-y,-x)} .
$$

We prove that the quotient is $o(y)$. In the first term of the numerator, we have $\lim _{y \downarrow} m(-y$, $-x)=v^{\prime}(0)$ by continuous differentiability, and $\lim _{y \downarrow} m(-x, 0)=v^{\prime}(0)$ by differentiability. Therefore $\lim _{y \downarrow 0}(m(-y,-x)-m(-x, 0))=0$. Because of this, because $r$ is a constant, and because $0<x<y$, the first term in the numerator, $r x(m(-y,-x)-m(-x$, $0)$ ), is $o(y)$. In the second term, $\lim _{y \downarrow 0}(m(-Z-x,-Z)-m(-x, 0))=v^{\prime}(-Z)-v^{\prime}(0)$, i.e. the limit exists and is real-valued. Because $p$ tends to 0 as $y$ tends to $0, r p(m(-Z-x$, $-Z)-m(-x, 0))$ tends to 0 , hence its product with $x, \operatorname{rxp}(m(-Z-x,-Z)-m(-x, 0))$, is $o(y)$. Thus the numerator is $o(y)$. The denominator tends to the positive constant $v^{\prime}(0)$ by continuous differentiability, and therefore the quotient is $o(y)$. $\square$

Proof of theorem 3.1. This theorem is proved as Theorem 2.1, with the new interpretations for $p$ and $r$ as explained in the text. $\square$

Proof of theorem 3.2. This theorem is proved as Theorem 2.2, with the new interpretations for $p$ and $r$ as explained in the text. Note however that, in the formal statement of Theorem 2.2 displayed in the proof of the theorem, $p$ is taken variable and its convergence to 0 (implied by the assumed convergence to 0 of $y$ ) is considered. This variability of $p$ needs further commenting in the context of Theorem 3.2. Now $p$ is a decision weight, so we must now permit decision weights to approach 0 arbitrarily closely. In other words, hazardous events $H$ for which the decision weight $W(H)$ are positive but approach arbitrarily close to 0 must be conceivable. For instance, if $W(H)=f(P(H))$ where $P$ is a probability and $f$ is a probability transformation function, then continuity of $f$ at 0 is required.

Also the role of $r$ needs further comments. In the proof of Theorem 2.2, we assumed that, for $p$ tending to $0, r$ was kept fixed. This condition is satisfied in the stepwise approach to dynamic decisions if event $R$ is kept fixed, but is more problematic in the reduced approach. To have $r$ fixed there, we must assume that events $H$, for which $W(H)$ tends to 0 , are associated with events $R$ such that always $W(H R) / W(H)=r$. This requires variation in the events $R$ which is not a natural assumption. It is more natural to keep the event $R$ fixed and then permit variability of $r=W(H R) / W(H)$ for decision weights $p$ tending to 0 . That preferable approach we consider next. With $r$ varying between 0 and 1 , the terms $r x(m(-y,-x)-m(-x, 0))$ and $\operatorname{rxp}(m(-Z-x,-Z)-m(-x, 0))$ in the nominator displayed in the proof of Theorem 2.2 are still $o(y)$, and therefore

$$
x-(1-r) y=o(y)
$$


still holds. If $r$ converges to a limit $r^{\prime}$ as $p$ and $y$ tend to 0 , then also $x-\left(1-r^{\prime}\right) y=o(y)$ holds, as the added term $\left(r-r^{\prime}\right) y$ then is $o(y)$. Note that such convergence is realistic in the case of subproportionality of the weighting function $w$, meaning that $r=w\left(r^{*} p\right) / w(p)$ increases as $p$ decreases towards 0 , for any probability $r^{*}$. The derived limiting results formalize the claim of Theorem 3.2 for the reduced approach, where event $R$ is kept fixed and its "conditional" decision weight $r$ varies while $p$ and $y$ tend to 0 .

Remark A.3. The expected utility results of this paper can be extended to the case where the loss $Z$ is not fixed, but is itself a random variable. First assume that $-Z$ designates a probability distribution over an interval $\left[-Z^{\prime},-Z^{\prime \prime}\right]$ where we assume that $Z^{\prime \prime}>y$; note that we assume that instead of $Z$, this same conditional probability distribution is to be substituted at all occurrences, i.e. the probability distribution of the loss is independent of everything else. The expected utility of $-Z$ is $u(-y) / p$. For each $z, v(-Z-z)$ is replaced by the expected utility of $-Z-z, v^{\prime}(-Z-z)$ is replaced by $\lim _{\epsilon \rightarrow 0}(E U(-Z-z)-$ $E U(-Z-z-\epsilon)) / \epsilon$ which exists under moderate smoothness assumptions on $v$; the corresponding inequalities are inherited from concavity of $v$. In proofs, $m(-Z-z,-Z)$ is defined as $(E U(-Z)-E U(-Z-z)) / z$. With these modifications, Theorems 2.1 and 2.2 still hold true under appropriate smoothness conditions for $v$.

For Theorems 3.1 and 3.2, new complications arise if $Z$ is variable. Then the decisionweight distribution of $Z$ can be different in PI than in NI, and it will also vary for limiting $p$ as in Theorem 3.2. Hence there does not seem to be an easy analog to the expected utility analysis in this case.

Proof of theorem 3.3. We first derive an exact lower bound for the reservation price $x$.

Lemma A.4 (An exact lower bound for $x$ expressed in terms of $a>0$ and $b$ ). If $v$ is concave, then

$$
\begin{aligned}
x \geq(1-r) y & + \\
& -\left(\frac{b}{a}\right) r y\left(\frac{v^{\prime}(-y-b)}{v^{\prime}(0)}\right)+r y p-r y p\left(\frac{v^{\prime}(-Z-y)-v^{\prime}(-Z)}{v^{\prime}(0)}\right) .
\end{aligned}
$$

Proof. Subtracting the equality $p v(-Z-a)=v(-y-b)$ from the equality $p v(-Z)=$ $v(-y)$ gives

$$
p(v(-Z)-v(-Z-a))=(v(-y)-v(-y-b)) .
$$

If $v$ is concave then it follows that

$$
p a v^{\prime}(-Z) \leq b v^{\prime}(-y-b)
$$

hence 


$$
p\left(\frac{v^{\prime}(-Z)}{v^{\prime}(-y-b)}\right) \leq \frac{b}{a}
$$

For later purpose we rewrite this as:

$$
r y p\left(\frac{v^{\prime}(-Z)}{v^{\prime}(-y-b)}\right) \leq r y \frac{b}{a}
$$

implying

$$
r y p\left(\frac{v^{\prime}(-Z)}{v^{\prime}(0)}\right) \leq r y \frac{b}{a}\left(\frac{v^{\prime}(-y-b)}{v^{\prime}(0)}\right)
$$

i.e.

$$
r y p\left(\frac{v^{\prime}(-Z-y)}{v^{\prime}(0)}\right) \leq r y \frac{b}{a}\left(\frac{v^{\prime}(-y-b)}{v^{\prime}(0)}\right)+\operatorname{ryp}\left(\frac{v^{\prime}(-Z-y)-v^{\prime}(-Z)}{v^{\prime}(0)}\right),
$$

hence

$$
r y p\left(\frac{v^{\prime}(-Z-y)}{v^{\prime}(0)}-1\right) \leq r y \frac{b}{a}\left(\frac{v^{\prime}(-y-b)}{v^{\prime}(0)}\right)+\operatorname{ryp}\left(\frac{v^{\prime}(-Z-y)-v^{\prime}(-Z)}{v^{\prime}(0)}\right)-r y p .
$$

The lower bound of Theorem 2.1 is

$$
x-(1-r) y \geq-\operatorname{ryp}\left(\frac{v^{\prime}(-Z-y)}{v^{\prime}(0)}-1\right)
$$

substituting here the inequality derived above gives

$$
x-(1-r) y \geq r y p-r y \frac{b}{a}\left(\frac{v^{\prime}(-y-b)}{v^{\prime}(0)}\right)-r y p\left(\frac{v^{\prime}(-Z-y)-v^{\prime}(-Z)}{v^{\prime}(0)}\right) .
$$

Next we derive the approximate lower bound of Theorem 3.3. Again, we assume that $y \downarrow$ 0 for fixed $Z, r, a$, so that also $x$ and $p$ tend to $0 .{ }^{8}$ The comments to variability of $p$ in the proof of Theorem 3.2 also apply here. In particular, one can also assume that $r$ is variable and substitute its limit $r^{\prime}>0$ for $r$ below. As this point requires no substantial changes, we do not discuss it further.

Because $p$ tends to $0, b$ must also tend to 0 . Theorem 3.3 indicates a bound (b/a)ry for the deviation from the actuarially adjusted premium, therefore $(b / a) r y$ seems the relevant order of magnitude. A formal statement of the theorem is 
Proposition A.5. $x \geq(1-r) y-\left(\frac{b}{a}\right) r y+o\left(\frac{b}{a} r y\right)$. We next prove this proposition. Consider the second term, $\left[-(b / a) r y\left(v^{\prime}(-y-b) / v^{\prime}(0)\right)\right]$, in the lower bound for $x$ in Lemma A.4. By continuous differentiability of $v, v^{\prime}(-y-b)$ tends to $v^{\prime}(0)$, and therefore $v^{\prime}(-y-b) / v^{\prime}(0)$ tends to 1 . It follows that the second term is $-(b / a) r y+(o b / a) r y$. The third and fourth terms can be grouped as $\operatorname{ryp}\left(1-\left(v^{\prime}(-Z-y)-v^{\prime}(-Z)\right) / v^{\prime}(0)\right)$. If $y$ tends to 0 , then by continuity of $v^{\prime}$ so does the numerator and therefore the whole quotient. Therefore, for $y$ sufficiently small, the third and fourth term together are positive, and can be dropped from a lower bound. This completes the proof.

Finally, note that the second and fourth terms in the lower bound in Lemma A.4 can be large if $y+b$, or $y$, are not very small and the second derivative of $v$ is extreme around $-y$ or on $[-y,-Z-y]$. In such cases the approximate lower bound in Theorem 3.3 may not be a true lower bound.

\section{Acknowledgment}

George $\mathrm{Wu}$ gave highly valuable and accurate comments. This research was supported by Grant SBR-9408684 from the National Science Foundation.

\section{Notes}

1. The loss in Question A in Section 1 can be interpreted, more generally, as a probability distribution over the interval $[0, Z]$. Remark A.3 in the Appendix shows that variability of $Z$ does not affect the subsequent expected utility analysis.

2. The present definition of probabilistic insurance differs slightly from that used by Kahneman \& Tversky (1979) in which whenever $H$ occurs the consumer loses either $Z$ or $y$ depending on whether $R$ does or does not occur.

3. Formally, $x / y$ tends to $1-r$ (i.e., $x-(1-r) y$ is " $o(y)$ ") if $r$ and $Z$ are kept fixed, $y$ tends to 0 , and the equality $p v(-Z)=v(-y)$ is kept so that $p$ also tends to 0 . The appendix gives details.

4. To simplify the display, we did not normalize the utility functions to satisfy $v(0)=0$; this, of course, does not affect the results.

5. Because each of the prospects considered in this paper involves only two outcomes, both nonpositive, the separable version of Kahneman \& Tversky (1979) and the cumulative version of Tversky \& Kahneman (1992) yield identical results.

6. Given the minor impact of marginal utility, a utility function that is convex but not to an extreme degree would give similar results.

7. One can equivalently derive the first-order approximation from the initial assumption $p \downarrow 0$ instead of $y \downarrow$ 0 . Then $y \downarrow 0$ follows from the same equality.

8. Again, one could equivalently assume $p \downarrow 0$ and then derive $y \downarrow 0$. 


\section{References}

Borch, K. (1974). “The Mathematical Theory of Insurance.” Lexington, MA: Lexington Books.

Camerer, C.F., and T.-H. Ho. (1994). "Violations of the Betweenness Axiom and Nonlinearity in Probability," Journal of Risk and Uncertainty 8, 167-196.

Chew, S.H., and L.G. Epstein. (1989). "The Structure of Preferences and Attitudes towards the Timing of the Resolution of Uncertainty," International Economic Review 30, 103-117.

Kahneman, D., and A. Tversky. (1979). "Prospect Theory: An Analysis of Decision under Risk," Econometrica 47, 263-291.

Loomes, G., and R. Sugden. (1986). "Disappointment and Dynamic Consistency in Choice under Uncertainty," Review of Economic Studies 53, 271-282.

Luce, R.D., and D. von Winterfeldt. (1994). "What Common Ground Exists for Descriptive, Prescriptive and Normative Utility Theories," Management Science 40, 263-279.

Merton, R.L. (1971). "Optimum Consumption and Portfolio Rules in a Continuous-Time Model," Journal of Economic Theory 3, 373-413.

Merton, R.C. (1993). "Operation and Regulation in Financial Intermediation: A Functional Perspective." In P. Englund (Ed.), Operation and Regulation of Financial Markets, 17-68, The Economic Council, Stockholm.

Mossin, J. (1968). "Aspects of Rational Insurance Purchasing,” Journal of Political Economy 76, 553-568.

Prelec, D. (1995). "The Probability Weighting Function," Econometrica, forthcoming.

Quiggin, J. (1981). "Risk Perception and Risk Aversion among Australian Farmers," Australian Journal of Agricultural Economics 25, 160-169.

Schmeidler, D. (1989). "Subjective Probability and Expected Utility without Additivity," Econometrica 57, 571-587.

Segal, U. (1988). "Probabilistic Insurance and Anticipated Utility," Journal of Risk and Insurance 55, $287-297$.

Segal, U. (1990). "Two-Stage Lotteries without the Reduction Axiom," Econometrica 58, 349-377.

Segal, U., and A. Spivak (1990). "First-Order versus Second-Order Risk-Aversion," Journal of Economic Theory $51,111-125$.

Tversky, A., and D. Kahneman (1992). "Advances in Prospect Theory: Cumulative Representation of Uncertainty," Journal of Risk and Uncertainty 5, 297-323.

Tversky, A., and P.P. Wakker (1995). "Risk Attitudes and Decision Weights," Econometrica 63, 1255-1280.

Viscusi, W.K. (1995). "Government Action, Biases in Risk Perception, and Insurance Decisions," Geneva Papers in Risk and Insurance Theory 20, 93-110.

Wu, G., and R. Gonzalez (1996). "Curvature of the Probability Weighting Function," Management Science 42, 1676-1690. 